\title{
Altered Metabolic Profile of Blood Plasma in Patients with Celiac disease: In-Vitro Proton Magnetic Resonance Spectroscopy Study
}

\author{
Deepti Upadhyay ${ }^{1}$, Govind K Makharia ${ }^{2}$, Naranamangalam R. Jagannathan ${ }^{1}$, Uma Sharma, ${ }^{1, *}$ \\ ${ }^{1}$ Departments of NMR \& MRI Facility, All India Institute of Medical Sciences, New Delhi, India \\ ${ }^{2}$ Gastroenterology \& Human Nutrition, All India Institute of Medical Sciences, New Delhi, India \\ *Corresponding author: umasharma69@gmail.com
}

Received July 16, 2015; Revised August 16, 2015; Accepted September 02, 2015

\begin{abstract}
Present study investigated the metabolic profile of blood plasma of patients with Celiac disease (CeD) and healthy controls (HC) using in-vitro nuclear magnetic resonance (NMR) spectroscopy to get an insight into the metabolic abnormalities in $\mathrm{CeD}$ and to determine the biomarker/s, if any, for the diagnosis of $\mathrm{CeD}$. The concentration of metabolites was determined using one-dimensional NMR at $700 \mathrm{MHz}$. The blood plasma from patients with CeD showed significantly higher $(\mathrm{p}<0.05)$ concentrations of several metabolites such as alanine (Ala), glycine (Gly), acetate (Ace) and creatine (Cr) compared to the HC subjects. While, concentration of creatinine (Crn) was significantly lower $(\mathrm{p}<0.05)$ in blood plasma of $\mathrm{CeD}$ patients. These findings suggested that the gluten induced inflammation resulted in significant alterations in metabolic activity in CeD. Partial least square discriminant analysis (PLS-DA) clearly distinguished the two groups in separate clusters on the score plot of samples from CeD patients and HC. Our results provided an insight into the alterations in metabolic pathways in $\mathrm{CeD}$ and indicated that the NMR spectroscopy of blood plasma in a large cohort of $\mathrm{CeD}$ patients may aid in determining the non-invasive biomarker/s for the diagnosis of CeD.
\end{abstract}

Keywords: Celiac disease, MR spectroscopy, metabolic profile, metabonomics, blood plasma

Cite This Article: Deepti Upadhyay, Govind K Makharia, Naranamangalam R. Jagannathan, and Uma Sharma, "Altered Metabolic Profile of Blood Plasma in Patients with Celiac disease: In-Vitro Proton Magnetic Resonance Spectroscopy Study.” International Journal of Celiac Disease, vol. 3, no. 3 (2015): 98-101 doi: 10.12691/ijcd-3-3-8.

\section{Introduction}

Celiac disease (CeD) is a chronic gastrointestinal autoimmune disorder that specifically affects the small intestine. The dietary intake of gluten and related prolamines present in cereals like wheat, rye, and barley results in inflammation of small intestine that leads to villous atrophy in genetically susceptible individuals (HLA- DQ2 and HLA-DQ8) [1]. The disease presents with a wide range of clinical manifestations that include chronic diarrhoea, abdominal pain, weight loss, fatigue and anaemia. Besides, $\mathrm{CeD}$ is reported to be associated with a large number of extra-intestinal manifestations, such as bone fractures, infertility, neurological disorders, skin lesions and liver abnormalities $[2,3]$ which contribute to the complexity of diagnosis of $\mathrm{CeD}$.

The $\mathrm{CeD}$ is reported in both children and adults with a prevalence of approximately $1 \%$ in worldwide $[4,5]$. Both genetic and environmental factors play an important role in the pathogenesis of $\mathrm{CeD}$, consequently affecting the metabolism. Thus, metabonomics study may provide valuable information on biomarkers that could be used for the diagnosis of $\mathrm{CeD}$. Metabonomics study provides information on time related quantitative multiple metabolic changes that occur in response to environment, pathophysiological stimuli and genetic modulation by investigating the biological samples, such as blood plasma, urine, using analytical spectroscopic techniques like highresolution nuclear magnetic resonance (NMR) spectroscopy combined with multivariate statistical methods [6].

Recently, a few studies have documented the metabonomics of blood plasma and urine in $\mathrm{CeD}$ patients using NMR spectroscopy and multivariate analysis methods $[7,8,9,10]$. Our group reported the characteristic metabolic signatures of villous atrophy in the intestinal mucosal biopsies of patients with $\mathrm{CeD}$ and compared with the data of disease control subjects using proton $\left({ }^{1} \mathrm{H}\right)$ NMR spectroscopy [10]. However, till date no study has determined the absolute concentration of metabolites in the blood plasma of $\mathrm{CeD}$ patients. The concentration of metabolites and their analysis combined with multivariate statistical modelling may present a comprehensive view of the alteration of metabolic pathway.

Therefore, the objectives of this study were (a) to determine the absolute concentration of metabolites in the blood plasma from patients with $\mathrm{CeD}$ and the healthy controls (HC) using ${ }^{1} \mathrm{H}$ NMR spectroscopy and (b) 
analyze the data with multivariate analysis to determine biomarker/s which may be used for the diagnosis of $\mathrm{CeD}$ patients and (c) to obtain an insight into the altered CeD metabolism.

\section{Patients and Methods}

This study was carried out between August 2012 and November 2014. The Institute Ethics Committee approved the study and an informed consent was obtained from each participant.

\subsection{Patients and Controls}

Twenty treatment naïve patients with CeD (mean age $32.5 \pm 10.4$ yrs; female 11; male 9) were recruited from the Celiac Disease Clinic of our Institution. The diagnosis was based on the positive serology and confirmed by histological examination of small bowel biopsies taken from the second part of the duodenum. Marsh-Oberhuber classification was applied for gradation of mucosal changes.

Twenty healthy subjects (mean age 27.5 \pm 3.4 yrs; female 8; male 12) serologically tested as negative for anti-tissue transglutaminase antibodies (tTGA), served as a control group (HC).

\subsection{Sample Collection and Processing for NMR Spectroscopy}

Blood samples were collected from each subject in the morning pre-prandial after overnight fasting. Peripheral venous blood samples $(5 \mathrm{ml})$ were collected in a heparinized vacutainer and centrifuged at 2000 RCF for 10 minutes at $4^{\circ} \mathrm{C}$. Then plasma was separated and stored at $-80^{\circ} \mathrm{C}$ until NMR experiment were carried out. For NMR spectroscopy, deuterium oxide $\left(\mathrm{D}_{2} \mathrm{O}\right), 0.5 \mathrm{mM}$ of sodium trimethyl-silyl-[2, 2, 3, 3- $\left.\mathrm{H}_{4}\right]$ propionate (TSP) and $0.5 \mathrm{mM}$ of sodium formate were added to the blood plasma samples. TSP served as a chemical shift reference while formate was used as concentration standard for the ${ }^{1} \mathrm{H}$ NMR studies.

\subsection{NMR Spectroscopy}

Proton NMR spectroscopy of the blood plasma samples was carried out on a narrow bore spectrometer operating at $700 \mathrm{MHz}$ (Agilent, U.S.A.) using one dimensional (1D) Carr-Purcell-Meiboom-Gill (CPMG) sequence with presaturation. The typical parameters for $1 \mathrm{D}$ experiment were: spectral width of $9000 \mathrm{~Hz}$; data points $32 \mathrm{~K}$; number of scans 64; spin echo delay, $\tau$ of $15 \mathrm{~ms}$ and a relaxation delay of 70s. A long relaxation delay was used, since formate typically has a long $\mathrm{T} 1$ relaxation time. Twodimensional (2D) total correlation spectroscopy (TOCSY) experiments were carried out for assignments of resonances.

The data was processed on a Dell 390N, PC, Red Hat Enterprise Linux workstation using the Varian software, Vnmrj 2.3 A. The free induction decays were multiplied by an exponentially decaying function prior to Fourier transformation. Spectra were manually phased using zero and first order corrections.

\subsection{Quantification of Metabolites}

The concentration of metabolites were determined by comparing the integrated intensity of isolated resonance of the compounds of interest with that of the intensity of the formate signal using the formula as presented earlier by us $[11,12,13]$.

\subsection{Statistical Analysis}

The concentration values of metabolites were reported as mean \pm standard deviation. Univariate analysis was carried out using SPSS software (SPSS version 11.5 for windows: SPSS, Inc., Chicago, IL). For comparison between the two groups, a Student's t-test/Wilcoxon Rank Sum test was used. A p-value $<0.05$ was considered as significant. Multivariate analysis was performed using Unscrambler 10.2 (Camo, Oslo, Norway). Principal component analysis (PCA) and Partial Least Square Discriminant Analysis (PLS-DA) were carried out on the concentration data of the metabolites. PCA is a powerful method of data extraction, which finds combinations of variables that describe trends in large data, called principal components and visualized in scores and loading plots. Score plots help in identifying clusters and outliers in the dataset where as loading plots show contributing variables to each principal component. PLS-DA shows the relationship between the spectra and the group information using multivariate regression methods. The metabolites responsible for the separation between the two groups are shown in loading plots.

Variables were mean centered and unit variance scaled and explored by PCA for initial analysis and detection of inherent trends and outlier detection. Further, a classification model was created from metabolites present in the blood plasma samples of patients with $\mathrm{CeD}$ and $\mathrm{HC}$ using PLS-DA.

\section{Results}

In all, 25 metabolites were assigned unambiguously in the blood plasma of patients with CeD using 1D and 2D TOCSY (spectrum not shown). The concentrations $[(\mu \mathrm{M} / \mathrm{L}$ (mean $\pm \mathrm{SD})]$ of metabolites were calculated from the blood plasma of CeD patients and $\mathrm{HC}$ and is presented as histogram in Figure 1.

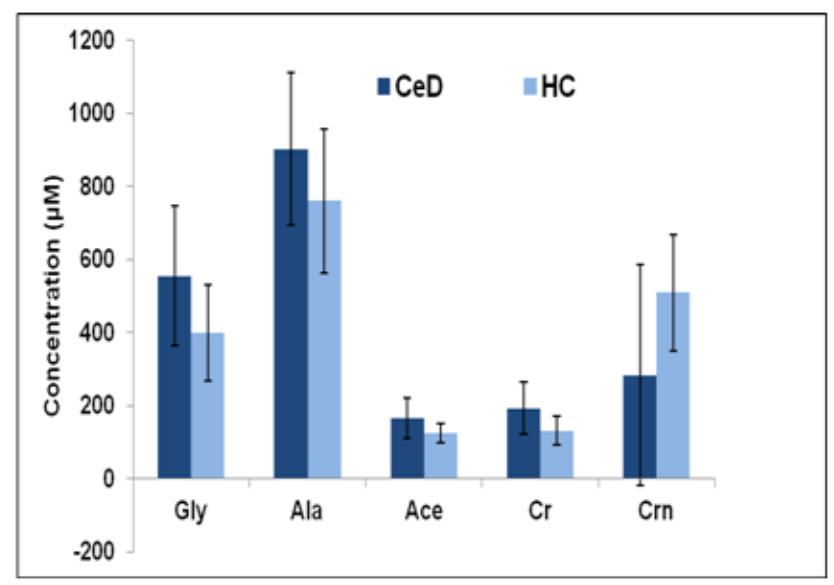

Figure 1. Histogram showing the comparison of concentration of metabolites between patients with $\mathrm{CeD}$ and $\mathrm{HC}$ 
Univariate analysis showed significantly higher $(\mathrm{p}<0.05)$ concentrations of alanine (Ala), glycine (Gly), acetate (Ace), and creatine (Cr) in patients with $\mathrm{CeD}$ in comparison to HC subjects. Whereas, a significantly lower $(p<0.05)$ concentration of creatinine (Crn) was observed in $\mathrm{CeD}$ patients as compared to the HC group.

Figure 2 shows a PLS-DA classification score plot that depicts the differentiation of the two groups; patients with $\mathrm{CeD}$ and the HC subjects in separate quadrants. The cross validated predicted fraction (Q2) value of the PLS-DA model for the blood plasma of $\mathrm{CeD}$ patients versus $\mathrm{HC}$ was 0.71 indicating the high reliability and strong predictive power of the PLS-DA model.

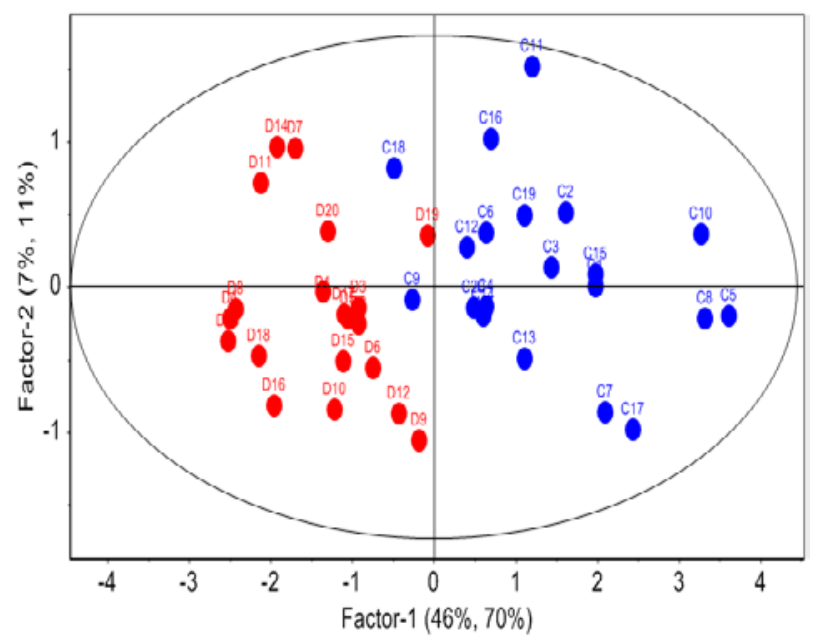

Figure 2. PLS-DA scores plot showing the separation between patients with $\mathrm{CeD}(\mathrm{D}, \bullet)$ and $\mathrm{HC}(\mathrm{C}, \bullet)$

\section{Discussion}

In the present study, ${ }^{1} \mathrm{H}$ NMR-based metabonomics approach applied on the blood plasma samples demonstrated the altered metabolic activity of the patients with CeD compared to the HC subjects.

Our data showed significantly higher concentration of Ace in CeD patients compared to that seen in HC subjects. Ace is an end product of the lipid metabolism, and its elevation in blood plasma may reflect increased utilization of lipids in order to meet energy requirements in $\mathrm{CeD}$ patients than in HC. Earlier study has reported the higher levels of glucose (Glc) and $\beta$-hydroxybutyrate in blood sera of CeD patients [7] and suggested that the ketone bodies are being used as energy source in the patients with CeD instead of Glc which is in agreement with our data. Since, the energy generation through these metabolic pathways is not as efficient as through the oxidation of Glc, it may be the metabolic basis of fatigue, which is usually experienced by the patients with CeD [7].

Our data also revealed a significantly higher concentration of gluconeogenic amino acids, namely, Gly and Ala in the blood plasma of patients with $\mathrm{CeD}$ than that observed in HC subjects. This indicated that the amino acids, Ala and Gly probably might not have been utilized for gluconeogenesis in CeD. Further, the utilization of ketone bodies has been shown to inhibit gluconeogenesis pathway which supports our findings [14].

Furthermore, our data showed significantly decreased concentration of Crn in the blood plasma of CeD patients as compared to the HC subjects. The low Crn levels in $\mathrm{CeD}$ patients may most likely be due to malnutrition as a consequence of mal-absorption [15]. In patients with $\mathrm{CeD}$, villous atrophy reduces the nutrients absorption, as a result, body needs an alternate energy source and it may utilize the fat and the protein present in the muscle tissue. This may probably leads to a significant loss of muscle mass and low Crn level observed in CeD patients [15]. Bertini et al. also reported significantly lower levels of amino acids (leucine, valine, proline, methionine) and Crn in blood plasma of $\mathrm{CeD}$ patients in comparison to controls [7]. Our results also revealed elevated levels of $\mathrm{Cr}$ in the blood plasma of CeD patients than healthy controls. Since, $\mathrm{Cr}$ is a prominent compound of metabolism which is involved in the energy supply for muscle metabolism, this imply that ATP production is altered in CeD patients which may lead to energy deficiency in $\mathrm{CeD}$ [16].

Further, multivariate analysis PLS-DA model clearly demonstrated significant biochemical dissimilarities in $\mathrm{CeD}$ patients and $\mathrm{HC}$ subjects and the samples from the two groups formed separate clusters on the score plot (Figure 2). The PLS-DA model was built with a significant predictive accuracy $(\mathrm{Q} 2=0.71)$. The loading plot provided the semi-quantitative information and depicted that several metabolites like tyrosine, choline, Ace and Crn contributed as discriminating markers between $\mathrm{CeD}$ patients and $\mathrm{HC}$ subjects.

\section{Conclusions}

The data showed that quantitation of metabolites provides an insight into altered metabolic pathways in $\mathrm{CeD}$ patients and showed that several metabolite may play an important role as biomarker/s for diagnosis of CeD, however, the study needs to be carried out in a large cohort of patients at different sites.

\section{Acknowledgements}

The Department of Biotechnology, Government of India is acknowledged for financial support (BT/BioCARe/01/233/2010-11).

\section{Conflict of Interest}

The authors have no conflicts of interest to disclose.

\section{References}

[1] Tye-Din, J. and Anderson, R., "Immunopathogenesis of celiac disease”, Curr Gastroenterol Rep, 10 (5), 458-65, Oct 2008.

[2] Farrell, R.J. and Kelly, C.P., "Celiac sprue”, N Engl J Med, 346 (3), 180-8, Jan 2002.

[3] Green, P.H., "The many faces of celiac disease: clinical presentation of celiac disease in the adult population”, Gastroenterology, 128 (4 suppl 1), S74-8, April 2005.

[4] Rewers, M., "Epidemiology of celiac disease: what are the prevalence, incidence, and progression of celiac disease?”, Gastroenterology, 128(4 Suppl 1), S47-51, Apr 2005.

[5] Makharia, G.K., Verma, A.K., Amarchand, R., Bhatnagar, S., Das, P., Goswami, A., Bhatia, V., Ahuja, V., Datta Gupta, S., Anand, K., "Prevalence of celiac disease in the northern part of India: A 
community based study”, J Gastroenterol Hepatol, 26 (5), 894900, May 2011.

[6] Nicholson, J., Lindon, J. and Holmes, E, "Metabonomics: understanding the metabolic responses of living systems to pathophysiological stimuli via multivariate statistical analysis of biological NMR spectroscopic data”, Xenobiotica, 29 (11), 1181-9, Nov 1999.

[7] Bertini, I., Calabro, A., Carli, V.D., Luchinat, C., Nepi, S., Porfirio, B., Renzi, D., Saccenti, E. and Tenori, L., "The Metabonomic Signature of Celiac Disease”, J Proteome Res, 8 (1), 170-7, Jan 2009.

[8] Bernini, P., Bertini, I., Calabrò, A., la Marca, G., Lami, G., Luchinat, C., Renzi, D., Tenori, L., "Are patients with potential celiac disease really potential? The answer of metabonomics", $J$ Proteome Res, 10 (2), 714-21, Feb 2011.

[9] Fathi, F., Ektefa, F., Arefi Oskouie, A., Rostami, K., RezaeiTavirani, M., Mohammad-Alizadeh, A.H., Tafazzoli, M. and Rostami Nejad, M., "NMR based metabonomics study on celiac disease in the blood serum", Gastroenterol Hepatol Bed Bench, 6 (4), 190-4, Autumn 2013.

[10] Sharma, U., Upadhyay, D., Mewar, S., Mishra, A., Das, P., Gupta, S.D., Dwivedi, S.N., Makharia, G.K. and Jagannathan, N.R., "Metabolic abnormalities of Gastrointestinal Mucosa in Celiac disease: An in-vitro proton NMR Spectroscopy Study”, J Gastroenterol Hepatol, April 2015.
[11] Sharma, U., Chaudhury, K., Jagannathan, N.R. and Guha, S.K.; "A proton NMR study of the effect of a new intravasal injectable male contraceptive RISUG on seminal plasma metabolites", Reproduction, 122 (3), 431-6, Sep 2001.

[12] Balasubramanian, K., Kumar, S., Singh, R.R., Sharma; U., Ahuja, V., Makharia, G.K. and Jagannathan N.R., "Metabolism of the colonic mucosa in patients with inflammatory bowel diseases: an in vitro proton magnetic resonance spectroscopy study”, Magn Reson Imaging, 27 (1), 79-86, Jan 2009.

[13] Sharma, U., Singh, R.R., Ahuja, V., Makharia, G.K. and Jagannathan, N.R., "Similarity in the metabolic profile in macroscopically involved and un-involved colonic mucosa in patients with inflammatory bowel disease: an in vitro proton ${ }^{1} \mathrm{H}$ MR Spectroscopy study”, Magn Reson Imaging, 28 (7), 1022-9, Sep 2010.

[14] Müller, M.J., Paschen, U. and Seitz, H. J., "Effect of ketone bodies on glucose production and utilization in the miniature pig", $J$ Clin Invest, 74 (1), 249-61, Jul 1984.

[15] Hari, P., Bagga, A., Mahajan, P. and Lakshmy, R.; "Effect of malnutrition on serum creatinine and cystatin C levels", $J$ Pediatr Nephrol, 22 (10), 1757-61, Oct 2007.

[16] Schicho, R., Nazyrova, A., Shaykhutdinov, R., Duggan, G., Vogel, H. and Storr, M., "Quantitative metabolomic profiling of serum and urine in DSS-induced ulcerative colitis of mice by ${ }^{1} \mathrm{H}$ NMR spectroscopy”, J Proteome Res, 9 (12), 6265-73, Dec 2010. 